\title{
Pattern Design of Ontology-based Knowledge Base for Project Risk Management
}

\author{
Chen-Jian HAO \\ School of Economics and Management \\ Xi'an Technological University \\ Xi'an, China \\ E-mail: hchj69@163.com
}

\begin{abstract}
This application of information system based on ontology knowledge model for the project risk management can effectively help the knowledge transfer and share with knowledge. On the basic of the research on relationship of knowledge model with knowledge ontology, the knowledge model sets up defined by three categories of domain knowledge, reasoning knowledge and task knowledge. On the basis of design pattern of the knowledge model, this paper put forward the structure of an applied ontology for the project risk management and its construction process.
\end{abstract}

Keywords-pattern design; ontology; knowledge base; project risk management

\section{INTRODUCTION}

The business enterprise internal knowledge can be shared by formal channel, network knowledge forum and teaching by personal example as well as verbal instrution. However, under the influence of employee's attitude and cognition, risk management knowledge is controlled in minority of hands. Therefore, in order to reduce the subjective of risk management knowledge share, it is necessary to build up more objective risk knowledge base system.

The knowledge model can conveniently describe knowledge by text form, but text form is disadvantageous to classification and logical reasoning. Knowledge ontology technology is used in the field of risk knowledge base system. It is an ideal approach to construct knowledge case based on ontology in order to store and represent domain knowledge.

\section{RELATIONSHIP BETWEEN KNOWLEDGE ONTOLOGY AND KNOWLEDGE MODEL}

\section{A. Structure of Knowledge Ontology}

As the view of ontology, the knowledge includes three parts: the logic, ontology and computing. The logic is the function that can deduce new logical representation from the present knowledge by logical computing. The computing is a deducing process. The ontology is a common, shared and formal description for important concepts in a specific domain, which includes a specification of the terms used (terminology) and agreements that allow to determine their meaning, along with the possible inter-relationships between these terms, standing for" concepts". The knowledge ontology is defined as one triple:

$$
\mathrm{KO}=\{<\mathrm{KA}>,\langle\mathrm{P}>,\langle\mathrm{P} \cup \lambda \varepsilon>\}
$$

KA (Knowledge Atom) is knowledge atom that indicates the minimum knowledge unit such as axiom, concept and basic operation relationship etc. $\mathrm{R}$ (Relation) is one set that shows interaction of knowledge atom and influence of knowledge entity. Rule is some regulations or operation set that is generated by knowledge atom or knowledge entity.

\section{B. Knowledge Model Based on Knowledge Ontology}

The knowledge model itself is a logical tool that elucidates "knowledge-intensive information-process structure". The knowledge model consists of three parts, one of which includes a group of related knowledge structure that is knowledge category.

Category one: domain knowledge that describes in detail information types in the application process of particular professional and technical knowledge.

Category two: logical reasoning knowledge that describes the basic logical reasoning step. The step is looked upon as the basic element of reasoning machine or inference engine.

Category three: task knowledge that describes the target of one application, sub-task and how to finish the target.

According to the classification of knowledge categories above, the knowledge model consists of three parts: domain knowledge model, reasoning or method knowledge model and task knowledge model. The relationship between knowledge model and knowledge ontology is shown in Figure 1.

Seeing from Figure 1, the solid arrow means logical elements of knowledge model and knowledge ontology respectively, and the dot arrow means that the elements of knowledge model can be represented by the elements of knowledge ontology respectively. The domain knowledge model and task knowledge model can be constructed by knowledge concept and relationship.

Seeing from Figure 1, the solid arrow means logical 
Figure 1. Relationship between knowledge model and knowledge ontology

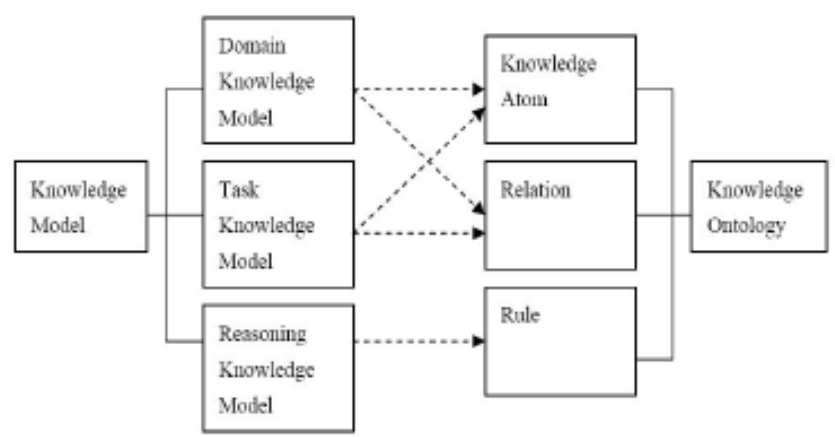

Elements of knowledge model and knowledge ontology respectively, and the dot arrow means that the elements of knowledge model can be represented by the elements of knowledge ontology respectively. The domain knowledge model and task knowledge model can be constructed by knowledge concept and relationship. The reasoning knowledge model is represented by rule of ontology. The knowledge model is described and represented by BNF (Backus - NaurForm) Paradigm. The knowledge framework is shown as follows:

- Knowledge model

$<$ Knowledge Model $>::=<$ Domain Knowledge $><$ Reason Knowledge $><$ Task Knowledge $>$

- Doman knowledge model

$<$ Domain Knowledge $>:=<$ Domain Pattern $><$ Use Base $>$

$<$ Domain Pattern $>\quad:=<$ Pattern Label $>$ $\{<$ Concept $>\mid<$ Relation $>\mid<$ Rule Type $>\}$

$<$ Pattern Label $>:=<$ Pattern Name $><[<$ Description $>]$

$<$ Concept $>::=[<$ Description $>]<$ Concept Name $>$ $[\{<$ attribute-list $>\}$

$\{<$ Subtype $>\} \mid<$ Aggregate $>\mid\{\langle$ Part $>\}]$ $\langle$ SupterConcept $>|$

$\langle$ Relation $\rangle:=\{\langle$ Binary Relation $\rangle[\langle$ Other Relation $\rangle]\}$

$<$ Binary Relation $>::=[<$ Description $>]<$ Relation

Name $><$ ParentRel $>$ "relation name" $<$ Child

Name $>$ "relation name" $<$ Inverse $>$ "relation

name" $[\{<$ attribute list $>\}]$

$<$ Other Relation $>::=<$ Binary Relation $>\{<$ Child elm>"relation name"\}

$<$ Rule Type $>:=<$ Rule Name $><$ Rule Define $>$

$<$ Rule Define $>\quad:=<$ Antecedent $><$ Consequent $><$ Symbol $>$ "Symbol isone of the operators"

$\langle$ Use Base $>:=<\{<$ Use $><$ Expressions $>\}$

$\langle$ Use $\rangle:=[\langle$ Description $\rangle]\langle$ Rule $\rangle\langle$ From $\rangle$

$<$ Expressions $>::=[<$ Description $>]<$ Expr Ante $><$ Expr Cons $><$ Expr Symbol $>$

- Reasoning knowledge model $\langle$ ReasonKnowledge $\rangle::=\langle$ Inference $\rangle\langle$ TransFunc $\rangle$

$\langle$ Inference $\rangle::=\langle$ Name $\rangle\langle$ InferRoles $\rangle\langle$ Specification $>$

$<$ InferRoles $>::=\langle$ Input $>\langle$ Output $>\langle$ Static $>$

$<$ TransFunc $>::=[<$ Description $>]<$ Name $><$ FuncType $><$ F Roles $>$ "refer to InferRoles"

$<$ FRoles $>::=<$ FInput $>$ "refer to Input" $<$ FOutput $>$ "refer to Output"

- Task knowledge model

$\langle$ TaskKnowledge $>::=\langle$ Task $>\langle$ Method $>$

$<$ Task $>::=\langle$ Name $><$ Goal $><$ TaskRoles $>$ "refer

to InferRoles" $[<$ Specification $>]$

$<$ TaskRoles $>::=\{<$ TaskInput $><$ TaskOutput $>\}$

$<$ Method $>:: \quad=<$ Name $\quad><$ Realizes $>\{<$ MethodRoles $>$

" refer to Infer-Roles" $<$ Decompositon $>$ "decompose to InferRoles and TransFun" $\}<$ ControlDesc $>$

\section{Design For Risk MANGMENT KNOWLEDGE-BASE SYSTEM}

\section{A. System Total Structure}

The structure of risk management knowledge-based system is made of four parts: the first part is database which is used to save the basic information of risk management, and relation database management base SQL Server is adopted because these information's are cases of knowledge Ontology. The second part is document base which is used to store common information to download, such as the risk management business models, solution, analysis report, standard norm and risk foundation knowledge, which is connected with database through ODBCFetch technology. The third is knowledge Ontology base which are presented by Formal languages OWL. The forth part is semantic logical rules of project risk management which are defined according to practice and saved by TXT text file. The system structure is showed in Figure 2.

Figure 2. System total structure

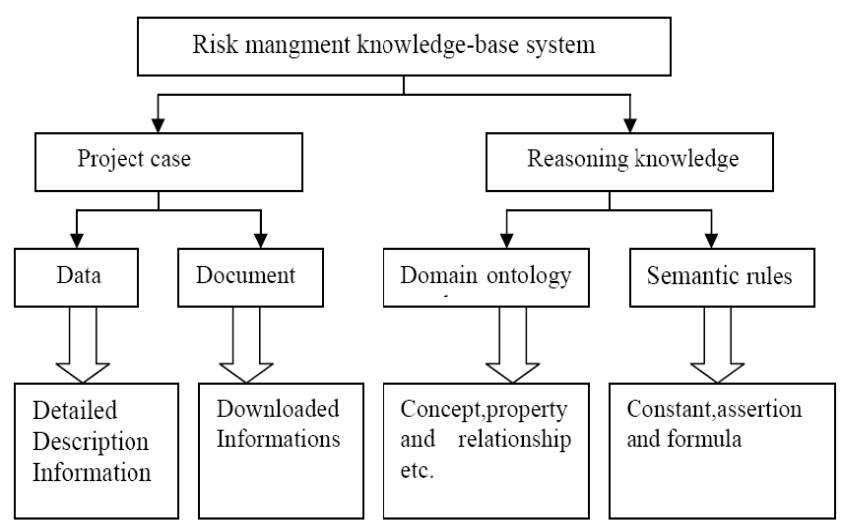




\section{B. The Construction Process for Knowledge Ontology of the Project Risk Management}

Referring to the Seven Approach developed by Stanford University, The basic construction process is set up as follows:

Step1: define the core application ontology of project risk management;

Step2: Make use of upper ontology Cyc system to ensure reasonable logic structure for the total application ontology system.

Step3: Set up the basic structure of the Class and reasoning mechanism through OpenCyc and make the presentation of project risk management concept through CycL.

Referring to" A Guide to the Project Management Body of Knowledge Body of Knowledge (PMBOK Guide-2000 Edition)" by Project Management Institute and "Introduction to Risk Management (Second Edition)" by Liu Jun, the knowledge ontology is divided into three hierarchies. The root node concept is project risk management, the top layer concept is five, one class concept is 12 , and the second class concept is 45.showed in Figure 3.

Figure 3. Ontology hierarchy

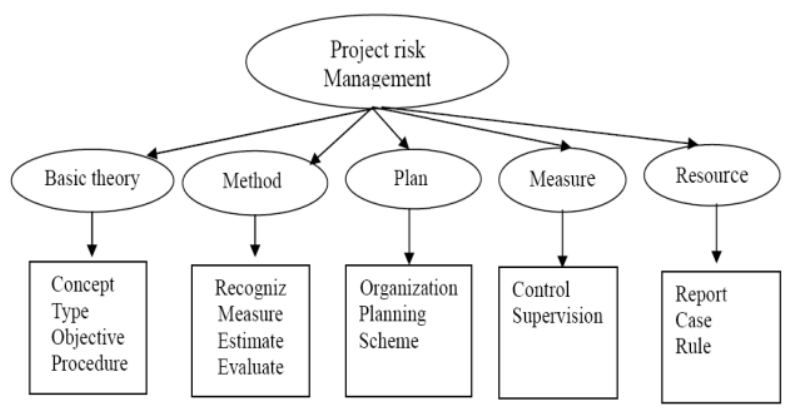

\section{The Design of Risk Project Case Database}

The information elements of knowledge-based system come from existent knowledge, rule and experience in each risk management process. Specifically, mainly include several types as follows:

- Basic information: Customer and representative director's information, project description, the whole risk grade and actual risk amount;

- Risk information: Risk category, serious grade, creation of probability, possible occurrence of time and actual occurrence of time;

- Risk strategy information: Strategy category and step, time of implementation, estimate cost, actual cost, related personnel, success or not etc.;

- Risk emergency measure information: Contingency plan, reason, time of implementation, estimate cost, actual cost, related personnel, success or not etc.; Those information are specific strategies;

- Field knowledge information: field expert, the risk category, strategy category, system of risk probability time frame system, risk expert's list, risk evaluation model and rules.

According to the analysis of the knowledge model and the knowledge base above, using the design pattern theory and software engineering theory for business process, the relationship chart set up in Figure 4.

- One manager manages several projects, one project generally has one main manager;

- One project exists an or several risks, one risk generally relates to one or several;

- One or several strategies can adopted to lower and decelerate risk, one strategy generally aims one particular risk;

- A strategy includes one or several emergency measures, one emergency measure generally is included in one strategy;

- One or Several field knowledge are used to estimate the risk category and strategy category;

- One expert can evaluate several projects; on project can be evaluated by several experts.

There are thirteen tables in this knowledge base. The first level has three tables, that is DomainBase, ReasonBase and TaskBase, and they corresponds to" knowledge model layer". The second level has six tables, that is usebase, modeltable, methodtable, plantable, resontable and transtable, and they correspond to "knowledge sub-model layer". The rest information table is the third level, and they correspond to "basic information layer".

Figure 4. Relationship chart

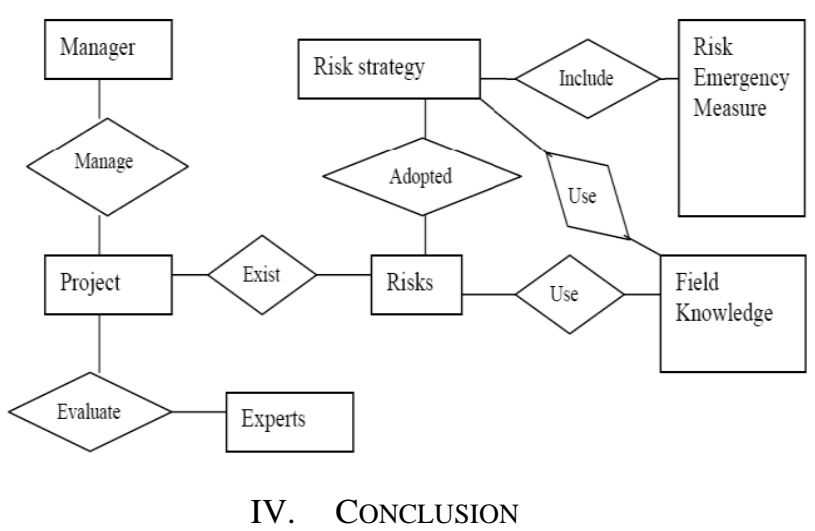

On the foundation of the research about knowledge model and knowledge base, the knowledge model based on ontology is set up. Aiming at the general process of project risk management, knowledge base system for project risk management is established according to design pattern by the use of knowledge ontology theory. For the concrete project risk management system or activity can be expanded on the basis knowledge system.

\section{ACKNOWLEDGEMENTS}

This work was supported by the National Science Foundation of China (No.71072158) and the President 
Foundation of Xi'an Technological University (No. XAGDXJJ1023).

\section{REFERENCES}

[1] Ling Qing-dong.Theory and Practice of Knowledge management. BeijingElectronics industrial publisher,2010.

[2] S. M. Deen, C. A. Johnson, "Formalizing an engineering approach to cooperating knowledge-based systems," in Knowledge and Data Engineering, IEEE Transactions on, 2006; 15( 1): pp.103-117.

[3] D. T. Pham, N. S. Gourash, "Knowledge-based configuration design," in Industrial Informatics, INDIN 2003 Proceedings, IEEE International Conference, 2003, pp.248-254.

[4] Project Management Institute. A Guide to the Project Management Body of Knowledge(PMBOK Guide-2000 Edition). Newton Square, PA: Project Management Institute, 2000

[5] Cf. T. R. Gruber, "A translation approach to portable ontologies," in Knowledge Acquisition, 1999, 5(2): pp.199-220. 